\title{
Black holes confirmed bald
}

Astrophys. J. Lett. 894, L1 (2020)

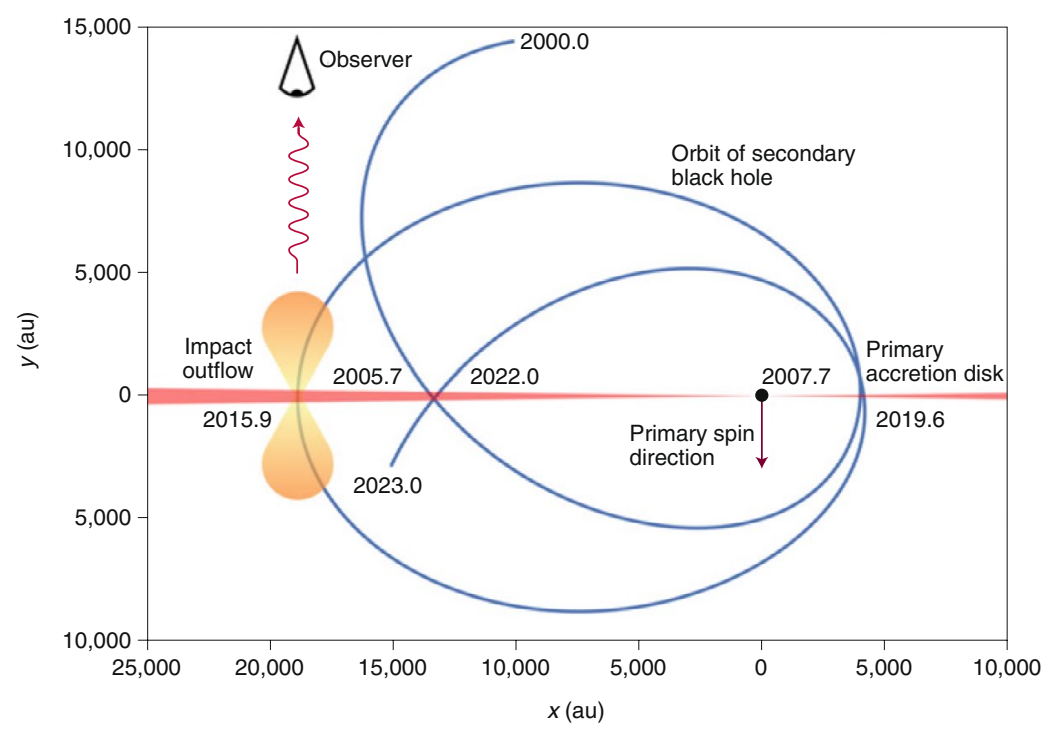

Credit: AAS

The recent detections of gravitational waves and the shadow of a black hole have led to unprecedented tests of general relativity (GR) and a renewed confidence in our physical understanding of the Universe. Seppo Laine and collaborators present observations of an infrared flare in the putative binary black hole system OJ 287 that allowed a further successful test of a different aspect of GR, the no-hair theorem: all black holes can be completely characterized by only three externally observable classical parameters, mass, electric charge and angular momentum.

OJ 287 is one of the most convincing cases of a galaxy hosting a supermassive black hole binary. With an optical lightcurve spanning more than 100 years, it has been found to flare periodically. The flares indicate that a secondary black hole is orbiting the primary on a highly eccentric orbit with a redshifted period of 12 years, plunging through the primary's accretion disk twice every orbit (pictured). These violent interactions create expanding bubbles of plasma, which give rise to thermal flares through brehmsstrahlung emission when they become optically thin.

Based on detailed GR calculations of the binary orbit that include contributions from higher multipoles due to gravitational wave emission and spin-orbit couplings, a flare had been predicted for 31 July 2019. Being hidden from Earth's view, OJ 287 was instead monitored by the Spitzer Space Telescope. Spectacularly, the source flared within the predicted four-hour window. Based on this validated prediction, Laine et al. were able to confirm the value of the dimensionless parameter $q$ (which relates the quadrupole moment and spin of the primary black hole) to be $1.00 \pm 0.15$. This value is in perfect agreement with the theoretical value of unity.

\section{Marios Karouzos}

Published online: 14 May 2020 https://doi.org/10.1038/s41550-020-1121-4 